\title{
Workshop on Software Engineering for Large Scale Computing (SELSC)
}

\author{
Daniel Rodríguez ${ }^{1}$ and Roberto Ruiz ${ }^{2}$ \\ ${ }^{1}$ Computer Science Department, The University of Alcalá \\ Ctra. Barcelona km. 33.6. 28871 Alcalá de Henares (Madrid), Spain \\ daniel.rodriguezg@uah.es \\ ${ }^{2}$ Computer Science Department, Pablo de Olavide University \\ Ctra. Utrera km. 1, 41013 Sevilla, Spain \\ robertoruiz@upo.es
}

\section{Motivation}

Computational Science techniques are increasingly being applied in both research institutions and industry to for example, financial applications, bioinformatics applications, physical applications, data mining applications based on grid or clusters etc. As with other disciplines there are specific issues when planning and developing such applications. For example, those applications are generally distributed and based upon Grid environments or clusters with specific management and planning issues, design, testing, etc.

This workshop was created with the intention of analysing software engineering issues specific to these types of applications such as adaptation of processes for, planning, management, verification and validation, testing, quality measurement, etc.

Also, there is also a motivation for further research on the other direction, i.e., the application of computational intelligence techniques to software engineering issues as a result of the creation of large metrics databases collected from software projects. Examples of computational techniques applied to Software Engineering include genetic algorithms, Bayesian networks, system dynamics, visualization, search based software engineering etc. Many emergent issues are subject to research efforts combining both computational techniques and Software Engineering.

\section{Topics of Interest and Objectives}

Therefore, topics of interest of this workshop included:

- Software processes for computational science applications

- Analysis and development of computational applications

- Testing of computational applications

- Computational intelligence techniques applied to software engineering

- Mining software engineering repositories

- Management issues for handling large amount of data

- Execution of data mining algorithms on grid and cluster environments

- Etc. 
And the objectives included:

- To bring together researchers and practitioners from computational science and software engineers backgrounds

- To steer discussion and debate on various aspects and issues related to software engineering processes when applied to computational problems

- How to apply software engineering techniques to computational intelligence problems

- To open research directions that are deemed essential by the researchers in the field and industry

The 9 selected papers out of the 19 submitted cover these topics from both points of view, i.e., software engineering issues for large scale applications and using computational science for helping with software engineering issues.

\section{Program Committee and Acknowledgements}

The organisers of this workshop would like to thank the organisers of main ICCS conference for their support, specially, Dr G D Dick van Albada. Also thanks to all members of the programme committee for all their effort reviewing and improving the submitted papers:

- Javier Dolado - Basque Country University, Spain

- Mark Harman - King's College London, UK

- Rachel Harrison - Stratton Edge Consulting, UK

- Andreas Jedlitschka - Fraunhofer IESE, Germany

- Taghi M. Khoshgoftaar - Florida Atlantic University, USA

- Andres A. Folleco - Florida Atlantic University, USA

- Dietmar Pfahl - University of Calgary, Canada

- Marek Reformat - University of Alberta, Canada

- Daniel Rodríguez - Univ. of Alcalá, Spain

- Mercedes Ruiz - Univ. of Cádiz, Spain

- Roberto Ruiz - Univ. of Seville, Spain

- Manoranjan Satpathy - General Motors, India

- $\quad$ Florian Urmetzer - University of Reading, UK

- Christian Weihrauch - University of Reading, UK

- Andrea Weise - University of Reading, UK

Finally, this workshop was also supported by grant number CCG07-UAH-TIC1588 , jointly supported by the University of Alcalá and the autonomic community of Madrid (Comunidad Autónoma de Madrid). 